\title{
Mucin 1 promotes radioresistance in hepatocellular carcinoma cells through activation of JAK2/STAT3 signaling
}

\author{
FENG-TAO $\mathrm{YI}^{1}$ and QI-PING LU ${ }^{2}$ \\ Departments of ${ }^{1}$ Radiotherapy and ${ }^{2}$ General Surgery, Wuhan General Hospital of Guangzhou Command, \\ People's Liberation Army, Wuhan, Hubei 430070, P.R. China \\ Received March 30, 2016; Accepted July 14, 2017
}

DOI: $10.3892 / \mathrm{ol} .2017 .7119$

\begin{abstract}
Mucin 1 (MUC1) is aberrantly overexpressed in numerous human cancer types, including hepatocellular carcinoma (HCC) and contributes to chemoresistance of tumor cells. The aim of the present study was to evaluate the possible implication of MUC1 in radioresistance of HCC cells and the underlying mechanisms. It was demonstrated that MUC1 was significantly upregulated in HCC cells following irradiation exposure, which was coupled with increased phosphorylation of signal transducer and activator of transcription 3 (STAT3). Enforced expression of MUC1 significantly $(\mathrm{P}<0.05)$ promoted the clonogenic survival of HCC cells following irradiation compared with empty vector-transfected cells. MUC1 overexpression resulted in $>60 \%$ reduction in apoptosis induced by irradiation, as determined by Annexin-V/propidium iodide double staining and flow cytometry analysis. Furthermore, overexpression of MUC1 significantly $(\mathrm{P}<0.05)$ attenuated the activation of caspase-3 and poly (ADP-ribose) polymerase in response to irradiation exposure. Mechanistically, MUC1 inhibited irradiation-induced apoptosis through activation of janus kinase 2 (JAK2) and STAT3, and induction of anti-apoptotic proteins induced myeloid leukemia cell differentiation protein Mcl-1 (Mcl-1) and BCL2 like 1 (Bcl-xL). Small hairpin RNA-mediated knockdown of STAT3 or MUC1 resensitized MUC1-overexpressing cells to irradiation-induced apoptosis, which was accompanied by reduced expression of Bcl-xL and Mcl-1. Collectively, MUC1 contributes to radioresistance of HCC cells likely through activation of the JAK2/STAT3 signaling pathway and thus represents a potential target for improving radiotherapy against HCC.
\end{abstract}

Correspondence to: Dr Qi-Ping Lu, Department of General Surgery, Wuhan General Hospital of Guangzhou Command, People's Liberation Army, 627 Wuluo Road, Wuhan, Hubei 430070, P.R. China

E-mail: 3349460151@qq.com

Key words: apoptosis, hepatocellular carcinoma, Mucin 1, radioresistance, signal transducer and activator of transcription 3 signaling

\section{Introduction}

Hepatocellular carcinoma (HCC) ranks as the fifth most common cancer type and the third leading cause of cancer-associated mortality worldwide $(1,2)$. The majority of patients with HCC are diagnosed at an advanced disease stage and are not amenable to potentially curative therapies, including surgery, transplantation, and radiofrequency ablation (3). Several therapies, including transarterial chemoembolization and radiation therapy are currently available for patients with locally advanced HCC (4). With recent advances in radiotherapy technology, a substantial dose of radiation can be precisely delivered to tumors, thereby reducing adverse effects on the surrounding normal tissues. It has been reported that radiotherapy yields remarkable local tumor control and has a potential beneficial survival impact in well-selected patients with HCC (5). However, the emergence of radioresistant tumor cells commonly leads to therapeutic failure. Therefore, understanding the mechanism underlying the resistance of HCC cells to radiation is of importance in improving the efficacy of radiotherapy.

Mucin 1 (MUC1) is a heterodimeric epithelial cell glycoprotein that is aberrantly overexpressed in a range of human cancer types (6). Mucin 1 protein has been implicated in various aspects of tumor development, including angiogenesis (7), proliferation (8), migration (9), survival (10) and metastasis (11). Accumulating evidence indicates a causal association between MUC1 expression and chemoresistance of tumor cells $(12,13)$. For instance, Nath et al (12) reported that MUC1 contributes to drug resistance in pancreatic cancer cells through upregulation of multidrug resistance genes, including ATP binding cassette subfamily $\mathrm{C}$ member $(\mathrm{ABCC}) 1, \mathrm{ABCC} 3, \mathrm{ABCC} 5$ and $\mathrm{ABCB} 1$. Overexpression of the oncogenic MUC1-C subunit has been identified to confer tamoxifen resistance in MCF-7 breast cancer cells (13). However, few studies have addressed the role of MUC1 in the development of radioresistance of tumor cells.

MUC1 serves as an oncogene in HCC and has been demonstrated to facilitate $\mathrm{HCC}$ cell migration and invasion (9). In the present study, the possible implication of MUC1 in the radioresistance of HCC cells was evaluated. The janus kinase/signal transducer and activator of transcription (JAK/STAT) signaling pathway serves an essential role in the growth and survival of HCC (14). Inhibition of STAT3 signaling has been reported to 
enhance radiation-induced apoptosis in HCC cells (15). Hence, the association between MUC1 and JAK/STAT3 signaling in the regulation of HCC cell radiosensitivity was also assessed.

\section{Materials and methods}

Cell culture. The human HCC SMMC-7721 cell line was obtained from the Type Culture Collection of the Chinese Academy of Sciences (Shanghai, China), and cultured in Dulbecco's modified Eagle's medium supplemented with 10\% fetal bovine serum (Invitrogen; Thermo Fisher Scientific, Inc., Waltham, MA, USA), $100 \mu \mathrm{g} / \mathrm{ml}$ penicillin and $100 \mu \mathrm{g} / \mathrm{ml}$ streptomycin at $37^{\circ} \mathrm{C}$ in a humidified atmosphere with $5 \% \mathrm{CO}_{2}$.

Plasmid construct and short hairpin RNAs (shRNAs). A full-length human MUC1 cDNA (OriGene Technologies, Inc., Rockville, MD, USA) was amplified by polymerase chain reaction (PCR) using Taq polymerase (Promega Corporation, Madison, WI, USA). The PCR primers are as follows: Forward, 5'-ATGACACCGGGCACCCAGTCT-3' and reverse, 5'-GCTACAAGTTGGCAGAAGTG-3'. Thermocycler conditions were as follows: Initial denaturation at $95^{\circ} \mathrm{C}$ for $10 \mathrm{~min}$, then 32 cycles of denaturation at $95^{\circ} \mathrm{C}$ for $30 \mathrm{sec}$, annealing at $60^{\circ} \mathrm{C}$ for $45 \mathrm{sec}$ and extension at $72^{\circ} \mathrm{C}$ for $60 \mathrm{sec}$. PCR products were subcloned into the mammalian expression vector pcDNA3.1(+) (Invitrogen; Thermo Fisher Scientific, Inc.). The identity of the pcDNA3.1/MUC1 plasmid was confirmed by DNA sequencing. STAT3 shRNA, MUC1 shRNA and negative control shRNA were purchased from Santa Cruz Biotechnology, Inc. (Dallas, TX, USA).

Cell transfection. For overexpression of MUC1, cells were transfected with the pcDNA3.1/MUC1 plasmid $(0.4 \mu \mathrm{g})$ or empty vector $(0.4 \mu \mathrm{g})$ using Lipofectamine 2000 transfection reagent (Invitrogen; Thermo Fisher Scientific, Inc.) according to the manufacturer's protocol. Transfected cells were selected for 2 weeks in the presence of $0.8 \mathrm{mg} / \mathrm{ml}$ of G418 (Sigma-Aldrich; Merck KGaA, Darmstadt, Germany). For the knockdown experiments, MUC1-overexpressing cells were transfected with control shRNA, STAT3 shRNA or MUC1 shRNA ( $1 \mu \mathrm{g}$ of each shRNA) using the Lipofectamine 2000 transfection reagent. Cells were harvested for gene expression analysis or exposure to irradiation $24 \mathrm{~h}$ after transfection.

Irradiation treatment. Cells $\left(5 \times 10^{6}\right)$ plated in $60-\mathrm{mm}$ tissue culture dishes were cultured until $\sim 60 \%$ confluency was achieved and then irradiated with 2-10 Gy of X-rays at a dose rate of $0.7 \mathrm{~Gy} / \mathrm{min}$ using an X-ray generator (Precision X-Ray, Inc., North Branford, CT, USA). Following treatment for 1, 3 , $6,10,16$, and $20 \mathrm{~h}$, cells were collected for further analyses. Non-irradiated cells were used as controls.

Clonogenic survival assay. For the clonogenic assays, cells were exposed to different does of X-rays as aforementioned. Immediately following irradiation, the cells were trypsinized, counted and reseeded onto 6-well plates at a density of 800 cells/well. The cells were cultured for 14 days and stained with $0.05 \%$ crystal violet at room temperature for $30 \mathrm{~min}$ (Sigma-Aldrich; Merck KGaA). The number of colonies consisting of $\geq 50$ cells was counted. The clonogenic survival curves were constructed from three independent experiments.

Reverse transcription quantitative PCR (RT-qPCR) analysis. MUC1 mRNA levels were determined using RT-qPCR analysis, as described previously (16). Briefly, total RNA was extracted using TRIzol reagent (Invitrogen; Thermo Fisher Scientific, Inc.) and reverse transcribed to first-strand cDNA using a PrimeScript RT reagent kit (Takara Biotechnology Co., Ltd., Dalian, China). PCR amplifications were performed using an ABI 7900 TaqMan Sequence Detection system (Applied Biosystems; Thermo Fisher Scientific, Inc.). The primer sequences for MUC1 were as follows: Forward, 5'-TCA GCTTCTACTCTGGTGCACAA-3' and reverse, 5'-ATTGAG AATGGAGTGCTCTTGCT-3'. PCR amplification of human GAPDH was performed in parallel with the primers: forward, 5'-CGACCACTTTGTCAAGCTCA-3'and reverse, 5'-AGG GGTCTACATGGCAACTG-3'. The relative MUC1 mRNA expression was determined according to the $2^{-\Delta \Delta C q}$ method following normalization against GAPDH transcripts (17).

Western blot analysis. Cells were lysed in radioimmunoprecipitation assay buffer (Thermo Fisher Scientific, Inc.) containing a Complete Protease Inhibitor Cocktail (Roche Diagnostics, Indianapolis, IN, USA) and centrifuged at $12,000 \mathrm{x} \mathrm{g}$ for $15 \mathrm{~min}$ at $4^{\circ} \mathrm{C}$. Total protein concentrations were quantified using a Pierce BCA Protein Assay kit (Thermo Fisher Scientific, Inc.) The supernatants containing total cellular protein were subjected to 10 or $12 \%$ SDS-PAGE. Subsequently, the proteins were transferred to nitrocellulose membranes. Membranes were blocked with 5\% fat-free milk in Tris-buffered saline (pH 7.4) with $0.1 \%$ Tween 20 (TBST) for $30 \mathrm{~min}$ at room temperature and incubated with the primary antibodies listed below overnight at $4^{\circ} \mathrm{C}$. After washing with TBST, membranes were incubated with horseradish peroxidase-conjugated goat anti-mouse (cat. no., sc-2005; 1:4,000 dilution) or goat anti-rabbit IgG (cat. no., sc-2004; 1:4,000 dilution; Santa Cruz Biotechnology, Inc.) for $1 \mathrm{~h}$ at room temperature, and developed using a enhanced chemiluminescence kit (GE Healthcare, Chicago, IL, USA). Protein signals were quantified via densitometric analysis using Quantity One software (version 4.6.2; Bio-Rad Laboratories, Inc., Hercules, CA, USA). The primary antibodies (1:500 dilution) used are as follows: Mouse monoclonal anti-MUC1 (cat. no. 4538); mouse monoclonal anti-phospho-STAT3 (cat. no. 4113); mouse monoclonal anti-STAT3 (cat. no. 9139); rabbit monoclonal anti- $\beta$-actin (cat. no. 4970); rabbit polyclonal anti-phospho-JAK2 (cat. no. 3771); rabbit polyclonal anti-JAK2 (cat. no. 3230) (all from Cell Signaling Technology, Inc., Danvers, MA, USA); rabbit polyclonal anti-cleaved caspase-3 (cat. no. ab2302); rabbit monoclonal anti-cleaved poly (ADP-ribose) polymerase (PARP; cat. no. ab32064); mouse monoclonal anti-induced myeloid leukemia cell differentiation protein Mcl-1 (Mcl-1; cat. no. ab114016); and rabbit monoclonal anti-BCL2 like 1 (Bcl-xL; cat. no. ab32370) (all from Abcam, Cambridge, MA, USA).

Apoptosis detection using flow cytometry. Apoptosis was measured using the Annexin V-FITC Apoptosis Detection kit (Sigma-Aldrich; Merck KGaA) according to the manufacturer's 


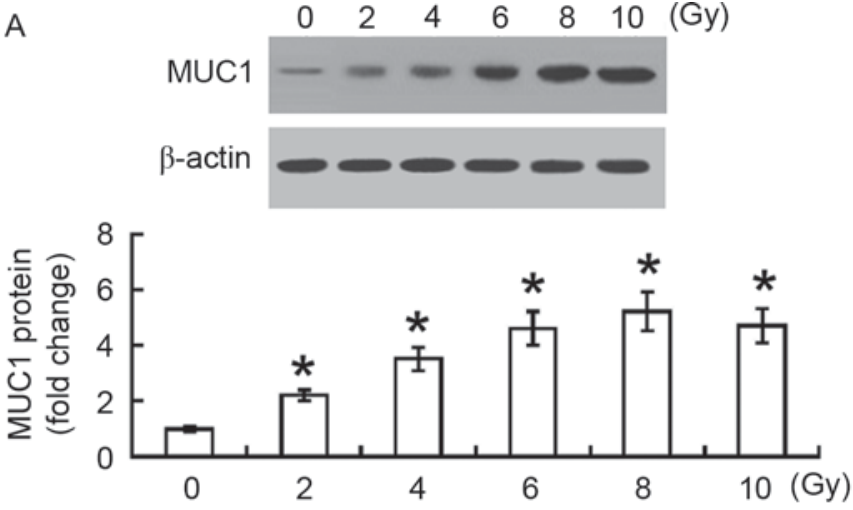

B
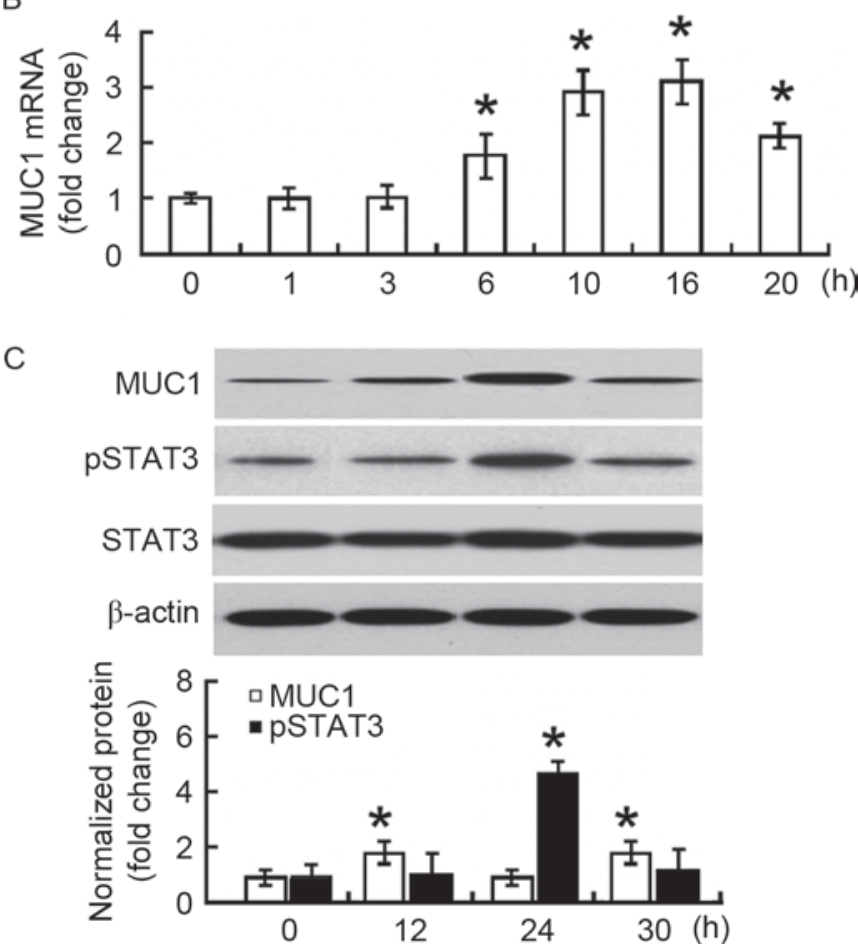

Figure 1. Irradiation induces the expression of MUC1 in hepatocellular carcinoma cells. (A) Western blot analysis of MUC1 protein in SMMC-7721 cells $24 \mathrm{~h}$ after exposure to indicated doses of irradiation. (B) Reverse transcription-quantitative polymerase chain reaction analysis of MUC1 mRNA abundance in SMMC-7721 cells exposed to 8 Gy irradiation and incubated for different times. (C) Western blot analysis of indicated proteins in SMMC-7721 cells exposed to 8 Gy irradiation for different times. Bar graphs present data from three independent experiments performed in triplicates. Data are expressed as the mean \pm standard deviation. " $\mathrm{P}<0.05$ vs. non-irradiated control. MUC1, Mucin 1; STAT3, signal transducer and activator of transcription 3; P, phosphorylated.

protocol. Briefly, cells were washed with Annexin V-binding buffer and incubated in binding buffer containing Annexin V-FITC $(25 \mu \mathrm{g} / \mathrm{ml})$ and propidium iodide (PI; $25 \mu \mathrm{g} / \mathrm{ml})$ for $10 \mathrm{~min}$ in the dark at room temperature. Stained cells were analyzed using a FacsCalibur flow cytometer (BD Biosciences, Franklin Lakes, NJ, USA).

Statistical analysis. Data are expressed as the mean \pm standard deviation. SPSS software version 16.0 (SPSS, Inc., Chicago, IL, USA) was employed to perform statistical analyses. Differences among multiple groups were analyzed by one-way analysis of variance followed by the Tukey's test. $\mathrm{P}<0.05$ was considered to indicate a statistically significant difference.

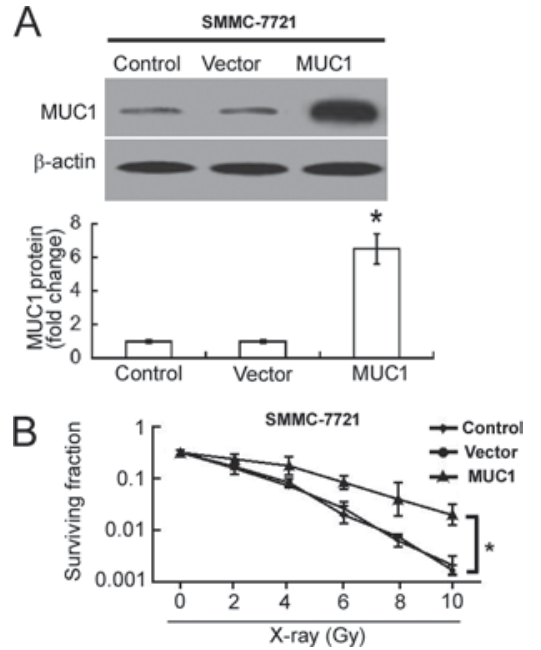

Figure 2. MUC1 overexpression decreases sensitivity of hepatocellular carcinoma cells to radiation. (A) Western blot analysis of MUC1 protein amounts in non-transfected cells and those transfected with MUC1-expressing plasmid or empty vector. (B) Clonogenic survival of SMMC-7721 cells exposed to different doses of X-rays. Compared with vector-transfected cells, MUC1-overexpressing cells demonstrated significantly higher clonogenic survival. Data are expressed as the mean \pm standard deviation. ${ }^{*} \mathrm{P}<0.05$ vs. vector-transfected cells. MUC1, Mucin 1.

\section{Results}

MUC1 is upregulated in HCC cells following irradiation. First, the changes in MUC1 protein expression in irradiated SMMC-7721 cells were measured. At $24 \mathrm{~h}$ after irradiation, a 2-5-fold increase in the MUC1 protein level was observed when compared with non-irradiated SMMC-7721 cells $(\mathrm{P}<0.05$; Fig. 1A). The increase was in a dose-dependent manner, reaching a peak at $8 \mathrm{~Gy}$. To confirm the upregulation of $\mathrm{MUC1}$ in response to irradiation, the changes in MUC1 mRNA abundance were examined. Time-course studies demonstrated that MUC1 mRNA levels increased and peaked at $16 \mathrm{~h}$ following 8 Gy irradiation (Fig. 1B). MUC1 protein levels reached a peak at $24 \mathrm{~h}$ after irradiation and then declined significantly at $30 \mathrm{~h}$ (Fig. 1C). Furthermore, phosphorylated STAT3 protein exhibited similar expression changes following irradiation (Fig. 1C).

MUC1 overexpression decreases the sensitivity of HCC cells to radiation. To determine the influence of MUC1 overexpression on cellular radiosensitivity, MUC1 was overexpressed in SMMC-7721 cells. Western blot analysis confirmed the upregulation of MUC1 in pcDNA3.1/MUC1-transfected cells compared with empty vector-transfected cells (Fig. 2A). Overexpression of MUC1 significantly increased the clonogenic survival of SMMC-7721 cells following irradiation when compared with empty vector-transfected cells $(\mathrm{P}<0.05$; Fig. 2B).

MUC1 overexpression inhibits irradiation-induced apoptosis. Next, the effect of MUC1 overexpression on radiation-induced apoptosis was examined using Annexin-V/PI staining analysis. Exposure to $8 \mathrm{~Gy}$ irradiation resulted in significant apoptosis in non-transfected and vector-transfected SMMC-7721 cells when compared with their corresponding non-irradiated controls ( $\mathrm{P}<0.05$; Fig. $3 \mathrm{~A})$. 

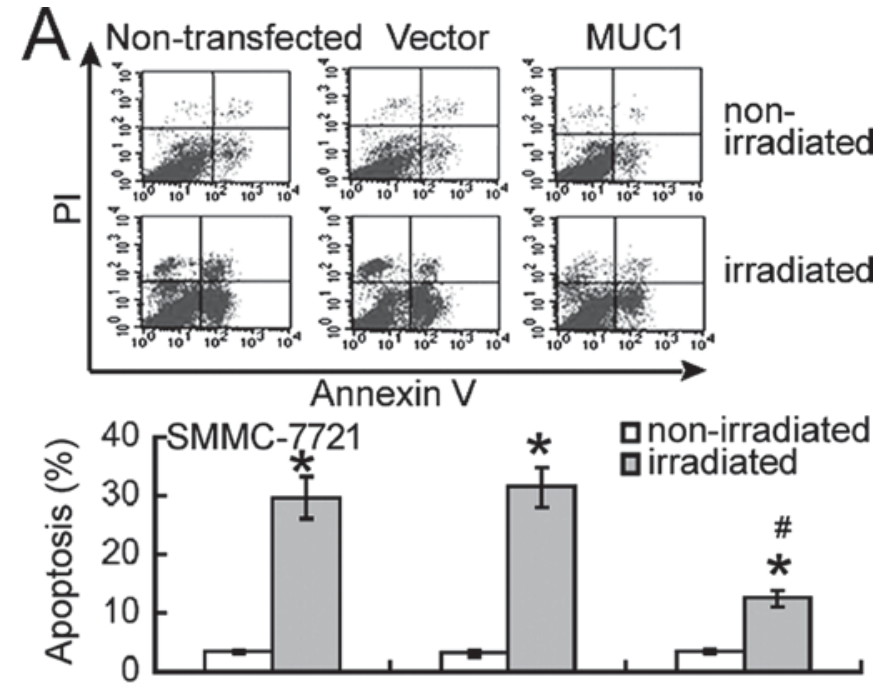

B

Non-

transfected Vector MUC1

irradiated

Cleaved caspase-3
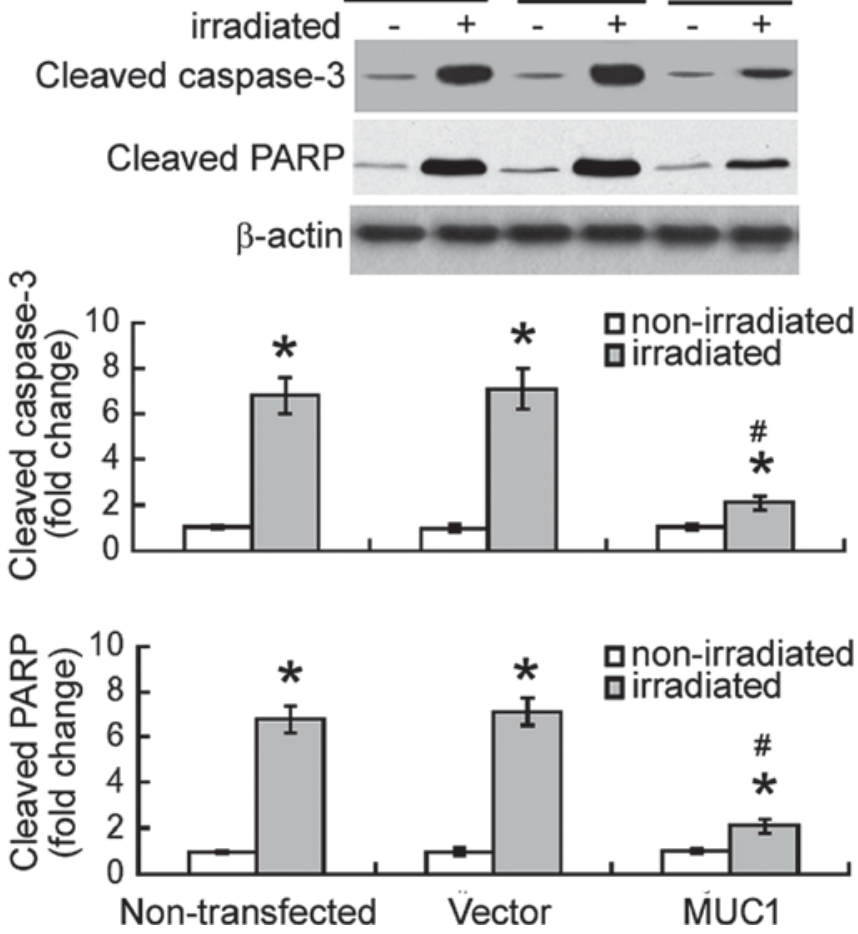

Figure 3. MUC1 overexpression suppresses irradiation-induced apoptosis (A) SMMC-7721 cells were exposed to 8 Gy irradiation. Following incubation for $48 \mathrm{~h}$, cells were stained with Annexin V and PI. The percentage of Annexin-V-positive apoptotic cells was determined. (B) Western blot analysis of the levels of cleaved caspase-3 and cleaved PARP in SMMC-7721 cells. Values are results from representative experiments performed in triplicates. Data are expressed as the mean \pm standard deviation. ${ }^{*} \mathrm{P}<0.05$ vs. non-irradiated counterparts; $\mathrm{P}<0.05$ vs. vector-transfected cells with irradiation. MUC1, Mucin 1; PARP, poly (ADP-ribose) polymerase; PI, propidium iodide.

Notably, enforced expression of MUC1 significantly inhibited the irradiation-induced apoptosis by $>60 \%(\mathrm{P}<0.05)$. Irradiation-induced apoptotic response was confirmed in SMMC-7721 cells by measuring the activation of caspase- 3 and PARP. It was observed that the levels of active cleaved caspase-3 and PARP were significantly increased in non-transfected and vector-transfected cells following irradiation $(\mathrm{P}<0.05$; Fig. 3B). Furthermore, overexpression of
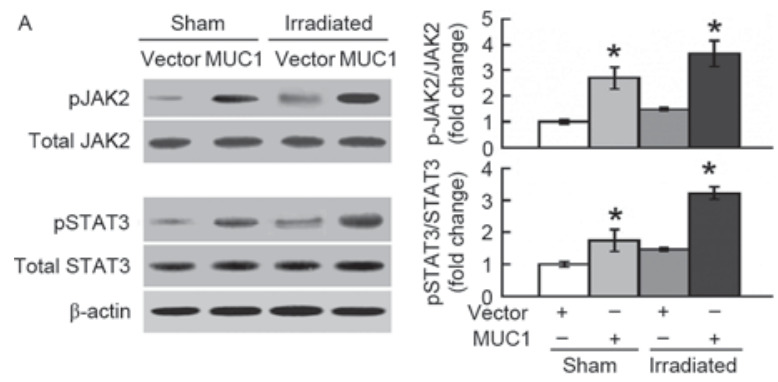

B
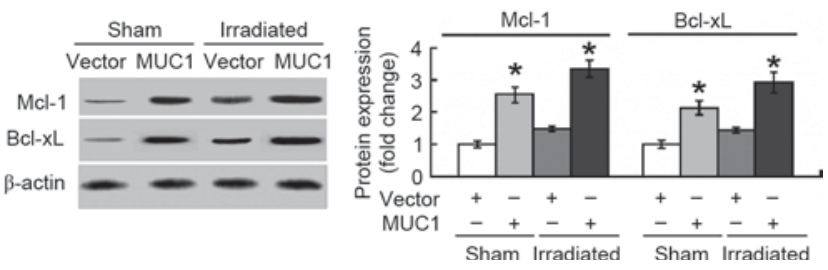

Figure 4. MUC1 overexpression activates JAK2/STAT3 signaling and upregulates Mcl-1 and Bcl-xL. Vector and MUC1 stably transfected SMMC-7721 cells were exposed to irradiation or sham-irradiated. Western blot analysis was performed to analyze the (A) phosphorylation of JAK2 and STAT3 and the (B) expression of Mcl-1 and Bcl-xL. Bar graphs present data from three independent experiments performed in triplicates. Data are expressed as the mean \pm standard deviation. ${ }^{*} \mathrm{P}<0.05$ vs. vector-transfected cells. MUC1 Mucin 1; STAT3, signal transducer and activator of transcription 3; JAK2, janus kinase 2; Mcl-1, induced myeloid leukemia cell differentiation protein Mcl-1; Bcl-xL, BCL2 like 1; P, phosphorylated.

A

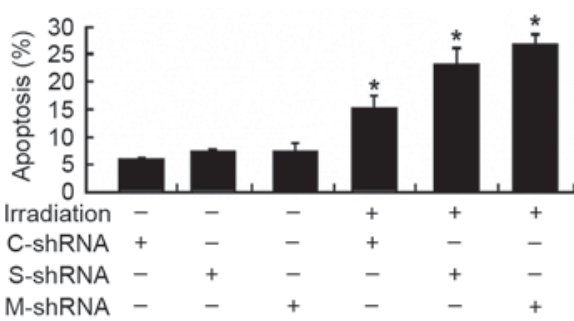

B
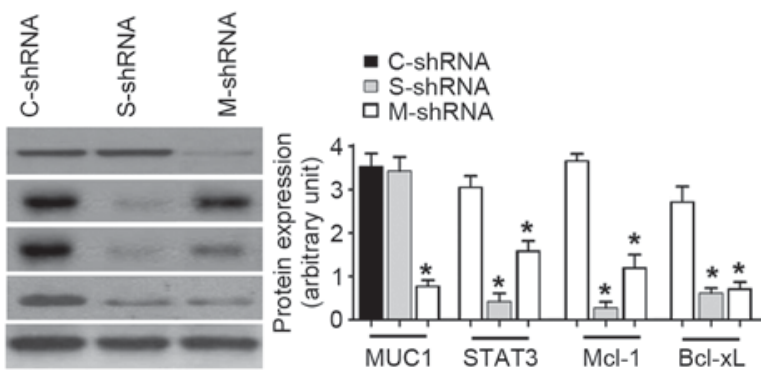

Figure 5. Knockdown of STAT3 or MUC1 enhances irradiation-induced apoptosis in MUC1 stably transfected SMMC-7721 cells. (A) Cells were transiently transfected with C-shRNA, S-shRNA or M-shRNA prior to irradiation exposure. Apoptotis analysis was determined by Annexin-V/propidium iodide staining. Values are results from representative experiments performed in triplicates. (B) Western blot analysis of indicated proteins in MUC1 stably transfected SMMC-7721 cells retransfected with C-shRNA, S-shRNA or M-shRNA. Left, representative western blots. Right, quantitative analysis of protein expression from three independent experiments. Data are expressed as the mean \pm standard deviation. ${ }^{*} \mathrm{P}<0.05$ vs. C-shRNA-transfected cells. MUC1, Mucin 1; STAT3, signal transducer and activator of transcription 3; Mcl-1, induced myeloid leukemia cell differentiation protein Mcl-1; Bcl-xL, BCL2 like 1; shRNA, short hairpin RNA; C-shRNA, control-shRNA; S, STAT3-shRNA; M-shRNA, MUC1 shRNA.

MUC1 significantly attenuated the activation of caspase-3 and PARP in response to irradiation exposure $(\mathrm{P}<0.05)$. 
MUC1 inhibits irradiation-induced apoptosis via activation of JAK2/STAT3. The molecular pathways involved in MUC1-mediated protection against apoptosis induced by irradiation were then explored. As presented in Fig. 4A, overexpression of MUC1 significantly promoted the phosphorylation of JAK2 and STAT3 in SMMC-7721 cells with or without irradiation treatment. In addition, the anti-apoptotic proteins Mcl-1 and Bcl-xL, downstream targets of STAT3, were induced by MUC1 overexpression (Fig. 4B).

To confirm the involvement of the STAT3 signaling pathway in the function of MUC1, MUC1-overexpressing cells were transfected with control or STAT3 shRNA prior to irradiation. Notably, the depletion of STAT3 restored the apoptotic response of MUC1-overexpressing cells following irradiation (Fig. 5A). Similarly, delivery of MUC1 shRNA resensitized MUC1-overexpressing cells to irradiation-induced apoptosis (Fig. 5A). At the molecular level, transfection with STAT3or MUC1-targeting shRNA significantly reduced the protein levels of Mcl-1 and Bcl-xL in MUC1 stably transfected SMMC-7721 cells (Fig. 5B).

\section{Discussion}

The data of the present study demonstrated that exposure to irradiation resulted in a transient increase in the mRNA and protein levels of MUC1 in HCC cells, suggesting its possible role as a stress-responsive survival factor. In support of this hypothesis, Chen et al (18) reported that MUC1 protects human colon cancer HCT116 cells from apoptosis under genotoxic stress induced by cisplatin. Similarly, Wei et al (19) demonstrated that MUC1 suppresses the p53-dependent apoptotic response to DNA damage. Under conditions of nutrient deprivation and hypoxia, MUC1 has been revealed to increase the cancer cell survival rate $(20,21)$. However, the adaptive induction of MUC1 in the present study did not appear to be sufficient to prevent apoptotic death in irradiated HCC cells, as significant apoptosis was detected at $48 \mathrm{~h}$ after irradiation. To confirm the role of MUC1 in the regulation of cellular response to irradiation, MUC1 was stably overexpressed in $\mathrm{HCC}$ cells and the clonogenic survival rate following irradiation exposure was examined. Of note, enforced expression of MUC1 significantly elevated the clonogenic survival of irradiation-treated HCC cells when compared with transfection of empty vector. Taken together, these results suggest that maintenance of high-level MUC1 protein is required to protect HCC cells from irradiation-induced apoptosis.

Induction of apoptosis is an important mechanism for killing cancer cells via radiation exposure (22). MUC1 has demonstrated the ability to modulate the apoptotic response in different cellular contexts. For instance, inhibition of MUC1 significantly induced apoptosis in pancreatic cancer cells (23) and increased the sensitivity of lung cancer cells to anticancer drug-induced apoptosis (24). Upregulation of MUC1 has been identified to render human bronchial epithelial cells more resistant to apoptosis following exposure to nickel acetate (25). Hence, the effect of MUC1 on radiation-induced apoptosis in HCC cells was further assessed in the current study. Notably, it was demonstrated that MUC1 overexpression significantly attenuated apoptotic response in irradiated HCC cells, as determined using Annexin-V/PI staining. Furthermore, irradiation-induced activation of caspase-3, a major mediator of apoptosis, was significantly compromised by MUC1 overexpression. These results collectively indicated that $\mathrm{MUC1}$ overexpression confers protection against radiation-induced apoptosis in HCC cells. However, one of the major limitations of the present study was the use of only one HCC cell line (SMMC-7721).

The JAK2/STAT3 signaling pathway is commonly associated with the emergence of apoptosis resistance in cancer cells, thus representing a potential target for anticancer therapy $(26,27)$. The data in the current study revealed that MUC1 overexpression significantly enhanced the phosphorylation of JAK2 and STAT3 in irradiated HCC cells. Furthermore, the downstream anti-apoptotic proteins $\mathrm{Mcl}-1$ and Bcl-xL were also induced by MUC1 overexpression. To check the possibility that MUC1 regulates the radiosensitivity of HCC cells through alteration of the JAK2/STAT3 signaling pathway, MUC1 and STAT3 shRNA were coexpressed prior to radiation treatment. Similar to the knockdown of MUC1, depletion of STAT3 reversed the protective effect of MUC1 against irradiation-induced apoptosis in HCC cells. Taken together, evidence was provided that MUC1-mediated radioresistance in HCC cells is partially ascribed to activation of the JAK2/STAT3 signaling pathway. Despite these findings, the involvement of other signaling pathways in the action of MUC1 cannot be excluded. Indeed, MUC1 has been documented to inhibit cisplatin-induced apoptosis of colon cancer cells via activation of JNK mitogen-activated protein kinase (MAPK) signaling (18). Modulation of extracellular-signal-regulated kinase 1/2, MAPK and Akt serine/threonine kinase signaling has also been reported to contribute to the oncogenic roles of MUC1 in pancreatic cancer cells (23).

In conclusion, to the best of our knowledge, the results of the present study have presented the first evidence for the implication of MUC1 overexpression in radioresistance of HCC cells. The results suggested that MUC1-mediated protection against irradiation-induced apoptosis is associated with activation of the JAK2/STAT3 signaling pathway, and induction of anti-apoptotic proteins Mcl-1 and Bcl-xL. Further studies are warranted to explore the significance of targeting MUC1 in improving radiotherapy in animal models of HCC.

\section{References}

1. Ferlay J, Soerjomataram I, Dikshit R, Eser S, Mathers C, Rebelo M, Parkin DM, Forman D and Bray F: Cancer incidence and mortality worldwide: Sources, methods and major patterns in GLOBOCAN 2012. Int J Cancer 136: E359-E386, 2015.

2. Forner A, Llovet JM and Bruix J: Hepatocellular carcinoma. Lancet 31: 1245-1255, 2012.

3. Schlachterman A, Craft WW Jr, Hilgenfeldt E, Mitra A and Cabrera R: Current and future treatments for hepatocellular carcinoma. World J Gastroenterol 21: 8478-8491, 2015.

4. Kalogeridi MA, Zygogianni A, Kyrgias G, Kouvaris J, Chatziioannou S, Kelekis $\mathrm{N}$ and Kouloulias V: Role of radiotherapy in the management of hepatocellular carcinoma: A systematic review. World J Hepatol 7: 101-112, 2015.

5. Lee IJ and Seong J: Radiotherapeutic strategies in the management of hepatocellular carcinoma. Oncology 81 (Suppl 1): S123-S133, 2011.

6. Nath S and Mukherjee P: MUC1: A multifaceted oncoprotein with a key role in cancer progression. Trends Mol Med 20: 332-342, 2014.

7. Kitamoto S, Yokoyama S, Higashi M, Yamada N, Takao S and Yonezawa S: MUC1 enhances hypoxia-driven angiogenesis through the regulation of multiple proangiogenic factors. Oncogene 32: 4614-4621, 2013. 
8. Gronnier C, Bruyère E, Lahdaoui F, Jonckheere N, Perrais M, Leteurtre E, Piessen G, Mariette C and Van Seuningen I: The MUC1 mucin regulates the tumorigenic properties of human esophageal adenocarcinomatous cells. Biochim Biophys Acta 1843: 2432-2437, 2014.

9. Wang J, Liu G, Li Q, Wang F, Xie F, Zhai R, Guo Y, Chen T, Zhang N, Ni W, et al: Mucin1 promotes the migration and invasion of hepatocellular carcinoma cells via JNK-mediated phosphorylation of Smad2 at the C-terminal and linker regions. Oncotarget 6: 19264-19278, 2015.

10. Zhao Q, Piyush T, Chen C, Hollingsworth MA, Hilkens J, Rhodes JM and Yu LG: MUC1 extracellular domain confers resistance of epithelial cancer cells to anoikis. Cell Death Dis 5: e1438, 2014.

11. Mohr AM, Bailey JM, Lewallen ME, Liu X, Radhakrishnan P, Yu F, Tapprich W and Hollingsworth MA: MUC1 regulates expression of multiple microRNAs involved in pancreatic tumor progression, including the miR-200c/141 cluster. PLoS One 8: e73306, 2013.

12. Nath S, Daneshvar K, Roy LD, Grover P, Kidiyoor A, Mosley L, Sahraei M and Mukherjee P: MUC1 induces drug resistance in pancreatic cancer cells via upregulation of multidrug resistance genes. Oncogenesis 2: e51, 2013.

13. Kharbanda A, Rajabi H, Jin C, Raina D and Kufe D: Oncogenic MUC1-C promotes tamoxifen resistance in human breast cancer. Mol Cancer Res 11: 714-723, 2013.

14. Zhou B, Chen H, Wei D, Kuang Y, Zhao X, Li G, Xie J and Chen P: A novel miR-219-SMC4-JAK2/Stat3 regulatory pathway in human hepatocellular carcinoma. J Exp Clin Cancer Res 33: $55,2014$.

15. Huang CY, Lin CS, Tai WT, Hsieh CY, Shiau CW, Cheng AL and Chen KF: Sorafenib enhances radiation-induced apoptosis in hepatocellular carcinoma by inhibiting STAT3. Int J Radiat Oncol Biol Phys 86: 456-462, 2013.

16. Li Y, Dinwiddie DL, Harrod KS, Jiang Y and Kim KC: Anti-inflammatory effect of MUC1 during respiratory syncytial virus infection of lung epithelial cells in vitro. Am J Physiol Lung Cell Mol Physiol 298: L558-L563, 2010.
17. Livak KJ and Schmittgen TD: Analysis of relative gene expression data using real-time quantitative PCR and the 2(-Delta Delta C(T)) Method. Methods 25: 402-408, 2001.

18. Chen Q, Li D, Ren J, Li C and Xiao ZX: MUC1 activates JNK1 and inhibits apoptosis under genotoxic stress. Biochem Biophys Res Commun 440: 179-183, 2013.

19. Wei X, Xu H and Kufe D: Human MUC1 oncoprotein regulates p53-responsive gene transcription in the genotoxic stress response. Cancer Cell 7: 167-178, 2005.

20. Yin L, Kharbanda S and Kufe D: Mucin 1 oncoprotein blocks hypoxia-inducible factor 1alpha activation in a survival response to hypoxia. J Biol Chem 282: 257-266, 2007.

21. Yin L, Kharbanda S and Kufe D: MUC1 oncoprotein promotes autophagy in a survival response to glucose deprivation. Int J Oncol 34: 1691-1699, 2009.

22. Balcer-Kubiczek EK: Apoptosis in radiation therapy: A double-edged sword. Exp Oncol 34: 277-285, 2012.

23. Tréhoux S, Duchêne B, Jonckheere N and Van Seuningen I: The MUC1 oncomucin regulates pancreatic cancer cell biological properties and chemoresistance. Implication of p42-44 MAPK, Akt, Bcl-2 and MMP13 pathways. Biochem Biophys Res Commun 456: 757-762, 2015.

24. Xu X, Wells A, Padilla MT, Kato K, Kim KC and Lin Y: A signaling pathway consisting of miR-551b, catalase and MUC1 contributes to acquired apoptosis resistance and chemoresistance. Carcinogenesis 35: 2457-2466, 2014.

25. Castorina A and Giunta S: Mucin 1 (MUC1) signalling contributes to increase the resistance to cell death in human bronchial epithelial cells exposed to nickel acetate. Biometals 27: 1149-1158, 2014.

26. Liu H, Tekle C, Chen YW, Kristian A, Zhao Y, Zhou M, Liu Z, Ding Y, Wang B, Mælandsmo GM, et al: B7-H3 silencing increases paclitaxel sensitivity by abrogating Jak2/Stat3 phosphorylation. Mol Cancer Ther 10: 960-971, 2011.

27. Lee DH, Sung KS, Bartlett DL, Kwon YT and Lee YJ: HSP90 inhibitor NVP-AUY922 enhances TRAIL-induced apoptosis by suppressing the JAK2-STAT3-Mcl-1 signal transduction pathway in colorectal cancer cells. Cell Signal 27: 293-305, 2015. 